\title{
A SIMPLE CONSTRUCTION FOR THE M-BAND DUAL-TREE COMPLEX WAVELET TRANSFORM
}

\author{
Ilker Bayram and Ivan W. Selesnick \\ Polytechnic University, Brooklyn, NY \\ ibayra01@utopia.poly.edu, selesi@poly.edu
}

\begin{abstract}
The dual-tree complex wavelet transform (DT- $\mathbb{C W T}$ ) which utilizes two 2-band discrete wavelet transforms (DWT) was recently extended to $M$-band by Chaux et al. In this paper, we provide a simple construction method for an $M$-band DT$\mathbb{C W T}$, with $M=r^{d}$ where $r, d \in \mathbb{Z}$. In particular, we show how to extend a given $r$-band DT-CWT to an $r^{d}$-band one. For convenience, the case where $r=2, d=2$ is considered. However, the scheme can be extended to general $\{r, d\}$ pairs straightforwardly. The extension to 2-D which achieves a directional analysis is also provided.
\end{abstract}

Index Terms - M-band dual-tree, 2-band dual-tree, Hilbert transform pairs, directional wavelets.

\section{INTRODUCTION}

The discrete dual-tree complex wavelet transform (DT- $\mathbb{C W T}$ ) [5] provides approximate shift-invariance and directional selectivity in 2D (and in higher dimensions). The DT- $\mathbb{C W T}$ achieves these properties by employing two discrete wavelet transforms (DWT) with the requirement that the wavelet associated with the second DWT is the Hilbert transform of the wavelet associated with the first DWT. The coefficients of the first and second DWT are then interpreted as the real and imaginary parts of a complex-valued wavelet transform. This scheme was extended to $M$-band orthonormal wavelet bases recently in [1], and used for image processing in [2]. The transform in $[1,2]$ employs two $M$-band discrete wavelet transforms where the wavelets associated with the two transforms form Hilbert transform pairs.

It can be shown for the 2-band case that, if the Hilbert transform relationship is required to be exact, the filters in one of the trees cannot be FIR if the other tree's filters are [11]. This is the same for the $M$-band case. To overcome this problem, in [1], the authors approximate the IIR filters using FIR filters by minimizing the $\mathbb{L}^{2}$ error of the frequency response and in [2] they perform the filtering operations in the frequency domain.

This work was supported by ONR under grant N00014-03-1-0217.
It is well known how to extend a 2-channel perfect reconstruction (PR) filter bank (FB) into an $M$-channel PR FB using a tree-structured FB (with $M=2^{k}$ ). A tree-structured FB also allows one to extend a 2-band DWT into an $M$ band DWT. $M$-band wavelet transforms of that type are often called wavelet packets [7]. However, it is not previously known how to properly extend a 2-band DT- $\mathbb{C W T}$ into an $M$ band one. This paper describes how to use a given $r$-band DT$\mathbb{C W T}$ to construct an $M$-band DT-CWT (with $M=r^{d}$ ). In particular, it will be shown how to obtain an FIR 4-band DT$\mathbb{C W T}$ from an FIR 2-band DT- $\mathbb{C W T}$ (for which several design methods are known). The construction can be extended to other $\{r, d\}$ values straightforwardly.

\section{THE DUAL-TREE WAVELET PACKET TRANSFORM}

It is well known that 2-band wavelet bases employ approximation spaces $V_{i}$ which can be decomposed into a higher level approximation space $V_{i+1}$ and a detail space $W_{i+1}$ as

$$
V_{i}=V_{i+1} \oplus W_{i+1}
$$

where $\oplus$ denotes a direct sum of the vector spaces. The 2 band dual-tree complex wavelet transform asks for a second set of approximation spaces $V_{i}^{\prime}$ and the associated orthogonal wavelet spaces $W_{i}^{\prime}$, such that the wavelets $\psi(t)$ and $\psi^{\prime}(t)$ form a Hilbert transform pair.

Similarly, the $M$-band wavelet transform employs approximation spaces $V_{i}$ satisfying $V_{i}=V_{i+1} \oplus W_{i+1}^{1} \oplus \ldots \oplus W_{i+1}^{M-1}$ [13]. The $M$-band DT- $\mathbb{C W T}$ is constructed $[1,2]$ by finding a second set of approximation spaces $V_{i}^{\prime}$ and wavelet spaces $W_{i}^{\prime k}$ such that the associated wavelet functions $\psi^{k}(t)$ and $\psi^{\prime k}(t)$ form Hilbert transform pairs, for $k \in\{1,2, \ldots, M-$ $1\}$. In the following, we will demonstrate how to construct an $r^{d}$-band DT-CWT given an $r$-band DT- $\mathbb{C W T}$. For convenience we will concentrate on the $\{r=2, d=2\}$ case, yielding a 4 -band DT- $\mathbb{C W T}$, but the procedure can be easily adapted to general $\{r, d\}$ pairs.

Suppose we are given a 2-channel orthonormal filter bank $\left\{h_{0}^{(2)}(n), h_{1}^{(2)}(n)\right\}$ and its associated scaling function $\phi^{(2)}(t)$ 


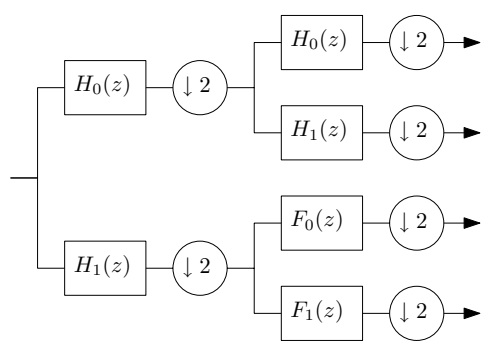

Fig. 1. A discrete wavelet packet transform.

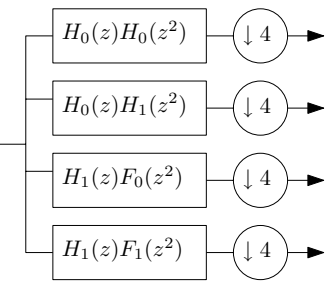

Fig. 2. Equivalent of the filter bank in Figure 1.

and wavelet $\psi^{(2)}(t)$, the Fourier transforms of which are defined by

$$
\begin{aligned}
& \Phi^{(2)}(\omega)=\prod_{l=1}^{\infty}\left[\frac{1}{\sqrt{2}} H_{0}^{(2)}\left(\frac{\omega}{2^{l}}\right)\right], \\
& \Psi^{(2)}(\omega)=\frac{1}{\sqrt{2}} H_{1}^{(2)}\left(\frac{\omega}{2}\right) \Phi^{(2)}\left(\frac{\omega}{2}\right) .
\end{aligned}
$$

Suppose we are also given a second 2-channel filter bank $\left\{h_{0}^{\prime(2)}(n), h_{1}^{\prime(2)}(n)\right\}$ and its associated scaling function $\phi^{\prime(2)}(t)$ and wavelet $\psi^{\prime(2)}(t)$, where $\psi^{\prime(2)}(t)$ is the Hilbert transform of $\psi^{(2)}(t)$, i.e.

$$
\Psi^{\prime(2)}(\omega)=j \operatorname{sgn}(\omega) \Psi^{(2)}(\omega)
$$

where 'sgn' denotes the signum function. That is, we are given a 2-band 'dual-tree' complex wavelet transform where the complex wavelet $\psi^{(2)}(t)+j \psi^{(2)}(t)$ is analytic.

Now we would like to construct a 4-band complex wavelet transform. To that end, suppose that $\left\{f_{0}(n), f_{1}(n)\right\}$ is another 2-channel orthonormal filter bank. We can then obtain a 4-channel orthonormal filter bank, namely a discrete wavelet packet transform [7], as illustrated in Figure 1. Our aim is to construct a second wavelet packet transform so that the wavelets (associated with the two wavelet packet transforms) form Hilbert transform pairs. Using noble identities, it can be seen that the filter bank in Figure 1 is equivalent to the filter bank in Figure 2. Now defining,

$$
\begin{aligned}
& H_{0}^{(4)}\left(e^{j \omega}\right):=H_{0}^{(2)}\left(e^{j \omega}\right) H_{0}^{(2)}\left(e^{j 2 \omega}\right), \\
& H_{1}^{(4)}\left(e^{j \omega}\right):=H_{0}^{(2)}\left(e^{j \omega}\right) H_{1}^{(2)}\left(e^{j 2 \omega}\right), \\
& H_{2}^{(4)}\left(e^{j \omega}\right):=H_{1}^{(2)}\left(e^{j \omega}\right) F_{0}\left(e^{j 2 \omega}\right), \\
& H_{3}^{(4)}\left(e^{j \omega}\right):=H_{1}^{(2)}\left(e^{j \omega}\right) F_{1}\left(e^{j 2 \omega}\right),
\end{aligned}
$$

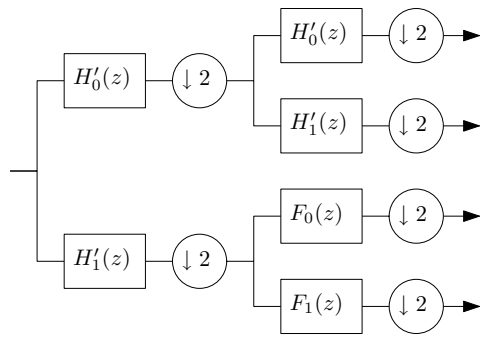

Fig. 3. The 'dual' of the tree in Figure 1.
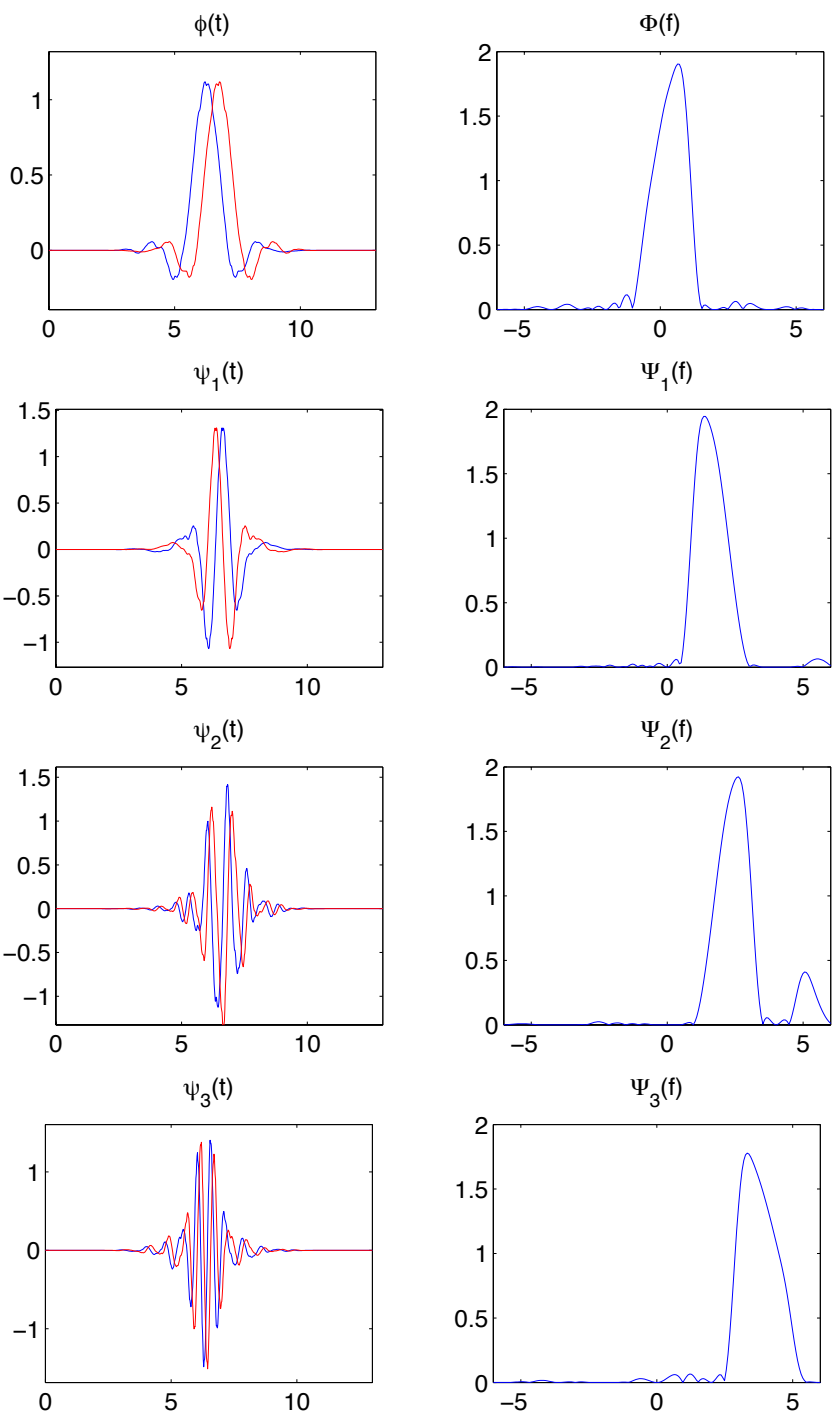

Fig. 4. The scaling functions, wavelets and the spectra of the complex functions for the 4-band dual-tree complex wavelet transform. Note that the wavelets are approximately analytic. Q-shift filters [6] of length 14 are used to produce these figures. 
we can show, using the infinite product formulas for the $M$ band $(M=4)$ case [13],

$$
\begin{aligned}
\Phi^{(4)}(\omega) & =\prod_{l=1}^{\infty}\left[\frac{1}{2} H_{0}^{(4)}\left(\frac{\omega}{4^{l}}\right)\right], \\
\Psi_{k}^{(4)}(\omega) & =\frac{1}{2} H_{k}^{(4)}\left(\frac{\omega}{4}\right) \Phi^{(4)}\left(\frac{\omega}{4}\right), \quad k \in\{1,2,3\},
\end{aligned}
$$

that the Fourier transforms of the scaling function and wavelets associated with this 4-channel filter bank can be written as,

$$
\begin{aligned}
& \Phi^{(4)}(\omega)=\Phi^{(2)}(\omega) \\
& \Psi_{1}^{(4)}(\omega)=\Psi^{(2)}(\omega) \\
& \Psi_{2}^{(4)}(\omega)=\frac{1}{\sqrt{2}} F_{0}\left(\frac{\omega}{2}\right) \Psi^{(2)}\left(\frac{\omega}{2}\right) \\
& \Psi_{3}^{(4)}(\omega)=\frac{1}{\sqrt{2}} F_{1}\left(\frac{\omega}{2}\right) \Psi^{(2)}\left(\frac{\omega}{2}\right) .
\end{aligned}
$$

Suppose now that we extend the second 2-channel filter bank similar to the first one as in Figure 1 but using a different 2-channel orthonormal filter bank $\left\{f_{0}^{\prime}(n), f_{1}^{\prime}(n)\right\}$. Then it follows as in (6) that,

$$
\begin{aligned}
\Phi^{\prime(4)}(\omega) & =\Phi^{\prime(2)}(\omega), \\
\Psi_{1}^{\prime(4)}(\omega) & =\Psi^{\prime(2)}(\omega), \\
\Psi_{2}^{\prime(4)}(\omega) & =\frac{1}{\sqrt{2}} F_{0}^{\prime}\left(\frac{\omega}{2}\right) \Psi^{\prime(2)}\left(\frac{\omega}{2}\right), \\
\Psi_{3}^{\prime(4)}(\omega) & =\frac{1}{\sqrt{2}} F_{1}^{\prime}\left(\frac{\omega}{2}\right) \Psi^{\prime(2)}\left(\frac{\omega}{2}\right) .
\end{aligned}
$$

Using (3), we can then write

$$
\begin{aligned}
& \Psi_{1}^{\prime(4)}(\omega)=j \operatorname{sgn}(\omega) \Psi_{1}^{(4)}(\omega), \\
& \Psi_{2}^{\prime(4)}(\omega)=j \operatorname{sgn}(\omega) \frac{F_{0}^{\prime}(\omega / 2)}{F_{0}(\omega / 2)} \Psi_{2}^{(4)}(\omega), \\
& \Psi_{3}^{\prime(4)}(\omega)=j \operatorname{sgn}(\omega) \frac{F_{1}^{\prime}(\omega / 2)}{F_{1}(\omega / 2)} \Psi_{3}^{(4)}(\omega) .
\end{aligned}
$$

Following this treatment, we conclude,

Theorem 1 Suppose we are given a Hilbert transform pair of wavelets $\psi(t), \psi^{\prime}(t)$ and a pair of CQFs $f_{k}(n), f_{k}^{\prime}(n)$ for $k \in\{0,1\}$. Let $\psi_{k}(t)$ be the wavelets obtained by decomposing $\psi(t)$ using $f_{k}(n)$ for $k \in\{0,1\}$. Also let $\psi_{k}^{\prime}(t)$ be the wavelets obtained by decomposing $\psi^{\prime}(t)$ using $f_{k}^{\prime}(n)$ for $k \in\{0,1\}$. Then $\psi_{k}(t)$ and $\psi_{k}^{\prime}(t)$ form Hilbert transform pairs if and only if $f_{k}(n)=f_{k}^{\prime}(n)$, for $k \in\{0,1\}$.

Thus, the new wavelets form Hilbert pairs if (and only if) we set $f_{k}^{\prime}(n)=f_{k}(n)$ for $k \in\{0,1\}$. Consequently, the 'dual' of the tree in Figure 1 is obtained by simply replacing $h_{k}(n)$ by $h_{k}^{\prime}(n)$ and leaving $f_{k}(n)$ the same, for $k \in\{0,1\}$ (see Figure 3).
This method generates a 4-band dual-tree complex wavelet transform. The resulting scaling functions, wavelets and the spectra of the resulting complex functions are illustrated in Figure 4. For these plots, q-shift filters [6] of length 14 are used as $h_{k}(n)$ and $h_{k}^{\prime}(n), f_{k}(n)$ is set equal to $h_{k}(n)$ for $k \in\{0,1\}$.

We note that this construction is different from that given in [3]. There, the authors use the dual-tree filters to decompose the detail spaces further while here the detail spaces are decomposed further using the same filters $f_{k}(n)$ in each of the two trees. The method of [3] results in the complex frequency responses of certain subbands not being analytic. Also, some of the associated wavelets will not be Hilbert transform pairs, which follows from the explanations above (see Figure 5).

The following section describes the implementation of the 4-band DT-CWT for discrete-time signals.

\section{THE DISCRETE-TIME IMPLEMENTATION OF THE 4-BAND DUAL-TREE COMPLEX WAVELET TRANSFORM}

The discrete-time implementation of the 2-band DT-CWT requires either that the first stage be different from the following stages or that specialized prefilters be used to generate the dual-tree filter bank input signals. Otherwise, the frequency responses of the first several stages of the DT- $\mathbb{C W T}$ are not single-sided as desired. It turns out that the same is true for the $M$-band case.

For the 2-band case, it was noted in [11] that if the first stage of the first FB consists of the filters $g_{k}(n)$, then it is required that the first stage of the second FB utilize $g_{k}(n-1)$ for $k \in\{0,1\}$. This modification can be derived by analyzing the FB responses and asking that the responses of the two FBs form discrete Hilbert transform pairs ${ }^{1}$. Following a similar analysis, for the 4-band case, we find the resulting first stage filters for the first tree as in Figure 6. The first stage of the dual-tree is obtained by replacing $g_{k}(n)$ by $g_{k}(n-1)$ and $h_{k}(n)$ by $h_{k}^{\prime}(n)$ for $k \in\{0,1\}$ as shown in Figure 7. Following the first stage, the low-pass branches in the two trees are iterated using the structure in Figure 1 and its dual (the structure in Figure 3).

We used the method described here to develop a 4-band DT-CWT based on FIR filters designed for the 2-band DT$\mathbb{C W T}$. The resulting complex frequency responses for the first two stages are shown in Figure 8.

Although the construction here differs from [3], if one restricts attention to a tree-structured filter bank with only two stages (i.e. Figures 6 and 7), then our construction is the same as that of [3].

\footnotetext{
${ }^{1}$ This is related but not equivalent to asking that the associated wavelets form Hilbert pairs.
} 

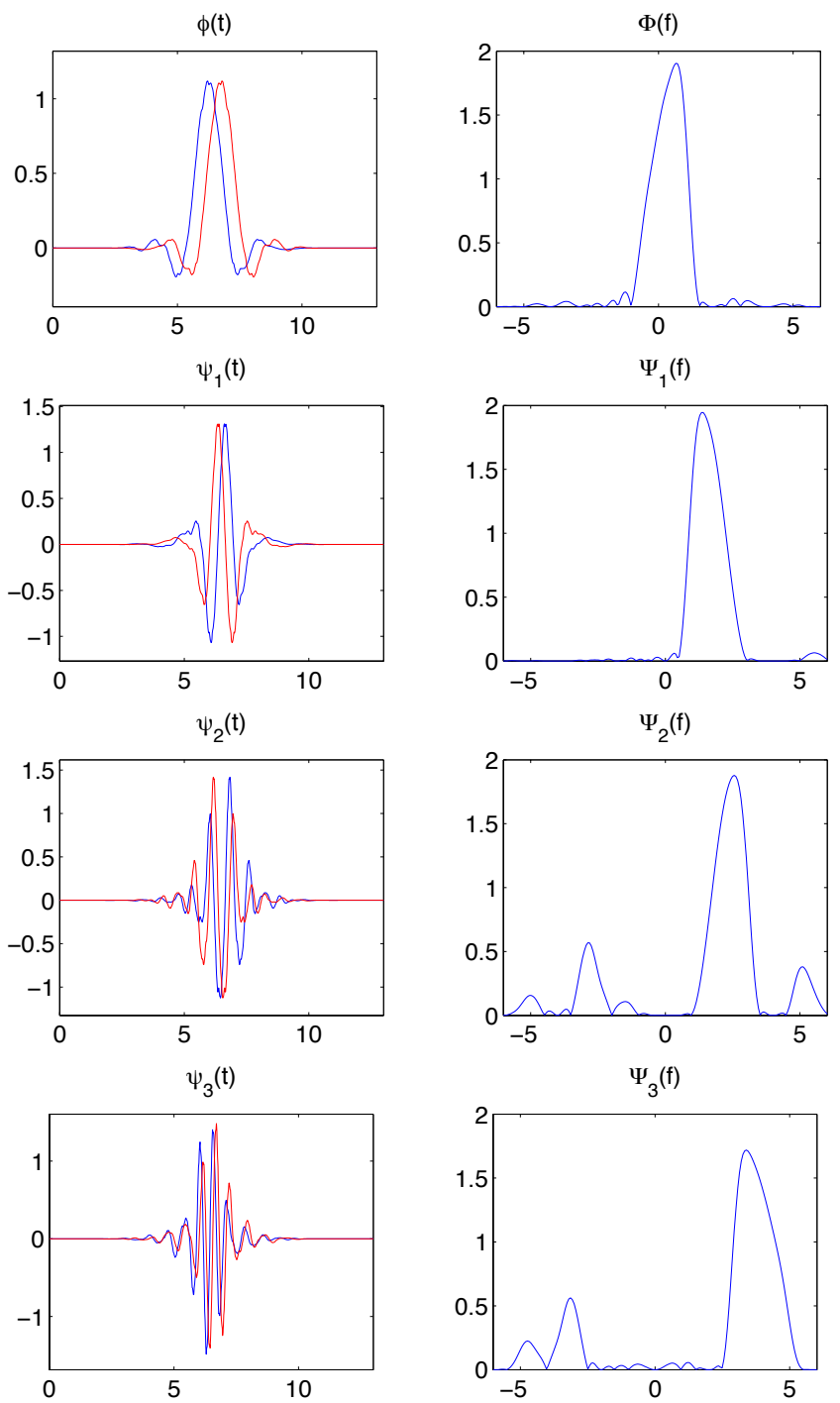

Fig. 5. The scaling functions, wavelets and the spectra of the complex functions for the 4-band dual-tree complex wavelet transform produced following the description in [3]. Note that two of the wavelets have significant energy on both positive and negative frequencies.

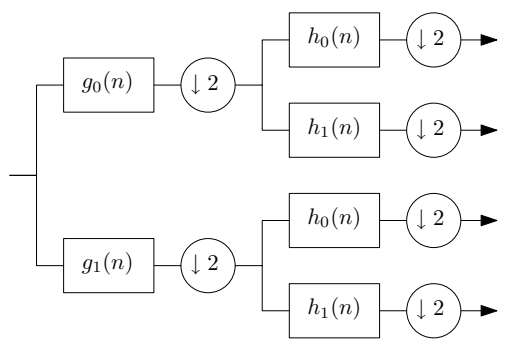

Fig. 6. The first stage of the first tree for the discrete-time implementation of the 4-band DT- $\mathbb{C W T}$.

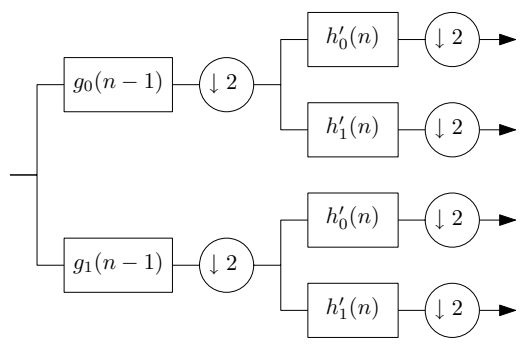

Fig. 7. The first stage of the second tree for the discrete-time implementation of the 4-band DT-CWT.
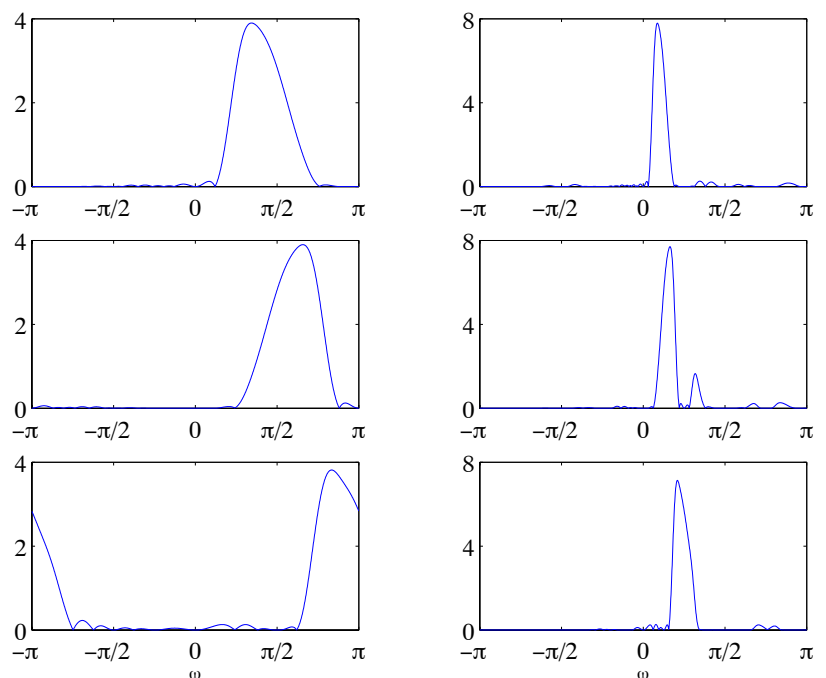

Fig. 8. The spectra of the responses of the complex transform for the first stage (left column) and the second stage (right column).

\section{SUFFICIENCY CONDITIONS FOR THE 4-BAND DT-CWT}

In [2], sufficient conditions are given for two $M$-band filter banks so that the associated wavelets form Hilbert transform pairs. The 4-band DT-CWT developed here satisfies these conditions. To verify that, we investigate the relationship among the filters in the 4-band filter bank. Using the necessary and sufficient conditions $[9,8]$ restricting the CQF FBs of an ideal DT-CWT for the 2-band case,

$$
\begin{aligned}
& H_{0}^{\prime(2)}\left(e^{j \omega}\right)=e^{-j 0.5 \omega} H_{0}^{(2)}\left(e^{j \omega}\right) \quad \text { for } \omega \in[0, \pi), \\
& H_{1}^{\prime(2)}\left(e^{j \omega}\right)=e^{-j(0.5 \pi-0.5 \omega)} H_{1}^{(2)}\left(e^{j \omega}\right) \text { for } \omega \in[0, \pi),
\end{aligned}
$$

and the definition of the filters in (4), we find that

$$
H_{k}^{\prime(4)}\left(e^{j \omega}\right)=e^{-j \theta_{k}(\omega)} H_{k}^{(4)}\left(e^{j \omega}\right)
$$


where (noting that $\theta_{k}(\omega)$ is an odd function)

$$
\theta_{0}(\omega)=\left\{\begin{aligned}
1.5 \omega & \text { if } \omega \in[0, \pi / 2) \\
1.5 \omega-\pi & \text { for } \omega \in[\pi / 2, \pi),
\end{aligned}\right.
$$

and

$$
\theta_{k}(\omega)=0.5 \pi-0.5 \omega \text { for } \omega \in[0, \pi), k \in\{1,2,3\}
$$

These are exactly the sufficiency conditions for Hilbert transform pairs of wavelets for the 4-band case, provided in [2]. Thus, we have verified our construction from another point of view.

Using FIR filters as explained, we obtained approximate $\theta_{k}(\omega)$ as shown in Figure 9. Note that $\theta_{2}(\omega)=\theta_{3}(\omega)$ as a consequence of our construction. For convenience, the underlying filter magnitude response is also shown. Observe that the approximations follow the ideal functions closely on the support of the underlying filter response.

\section{2-D 4-BAND DUAL-TREE TRANSFORM}

The 4-band DT-CWT may be extended to 2-D similar to the 2-band case [11]. First, the two transforms making up the dual-tree transforms are extended to 2-D similarly as any other separable wavelet transform. Then, denoting the 2-D transform of the first tree by $\mathbf{T}_{\mathbf{1}}$ and that of the dual-tree by $\mathbf{T}_{\mathbf{2}}$, the 2-D directional transform, following [11], is given by,

$$
\mathbf{T}_{\mathbf{2 D}}=\frac{1}{2}\left[\begin{array}{cc}
\mathbf{I} & -\mathbf{I} \\
\mathbf{I} & \mathbf{I}
\end{array}\right]\left[\begin{array}{c}
\mathbf{T}_{\mathbf{1}} \\
\mathbf{T}_{\mathbf{2}}
\end{array}\right]
$$

The resulting directional wavelets are illustrated in Figure 10.

This transform which may be referred to as a real oriented transform (following [11]) is $2 \mathrm{X}$ overcomplete. However, one can similarly construct a 2-D 4-band DT-CWT as explained in [11]. This would result in a transform that is $4 \mathrm{X}$ overcomplete.

\section{A COMPARISON WITH THE GENERAL CASE}

In general, a 4-band DT-CWT $[1,2]$ as mentioned in Section 2 , calls for approximation spaces $V_{i}, V_{i}^{\prime}$ which satisfy

$$
V_{i}=V_{i+1} \oplus W_{i+1}^{1} \oplus W_{i+1}^{2} \oplus W_{i+1}^{3}
$$

and

$$
V_{i}^{\prime}=V_{i+1}^{\prime} \oplus W_{i+1}^{\prime 1} \oplus W_{i+1}^{\prime 2} \oplus W_{i+1}^{\prime 3}
$$

such that $\psi_{i}^{k}(t)$ and $\psi_{i}^{\prime k}(t)$ form Hilbert transform pairs for $k \in\{1,2,3\}$.

In contrast, our approach is based on decomposing a 2band orthonormal wavelet basis. The approximation spaces for the 2-band basis $\hat{V}_{i}$, originally satisfy $\hat{V}_{i}=\hat{V}_{i+1} \oplus \hat{W}_{i+1}$. It is known that [7] iterating the high-pass branch as in Figure 1 corresponds to decomposing $\hat{W}_{i}$ as $\hat{W}_{i}=\hat{W}_{i}^{0} \oplus \hat{W}_{i}^{1}$ (note
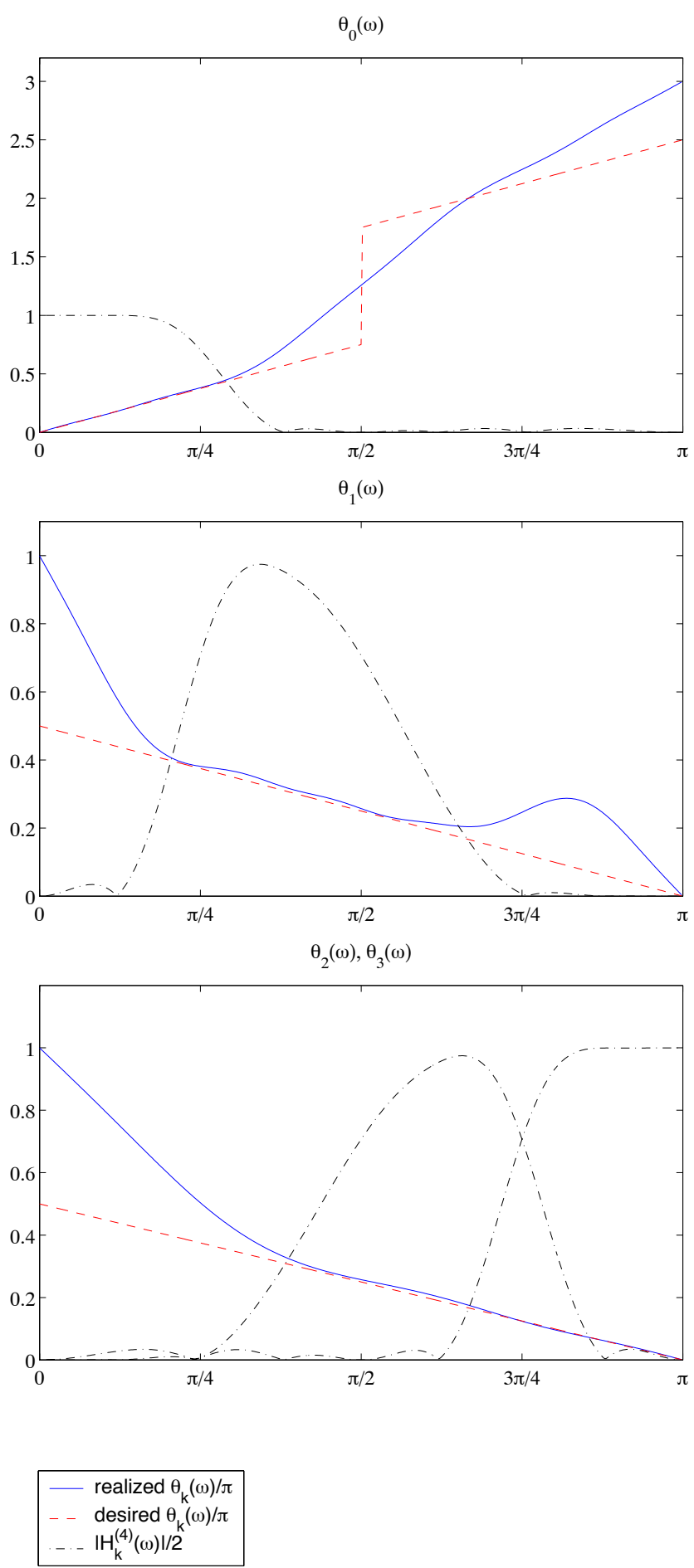

Fig. 9. $\theta_{k}(\omega)$ for $k \in\{0,1,2,3\}$. See (11), (12) for the desired $\theta_{k}(\omega)$. 

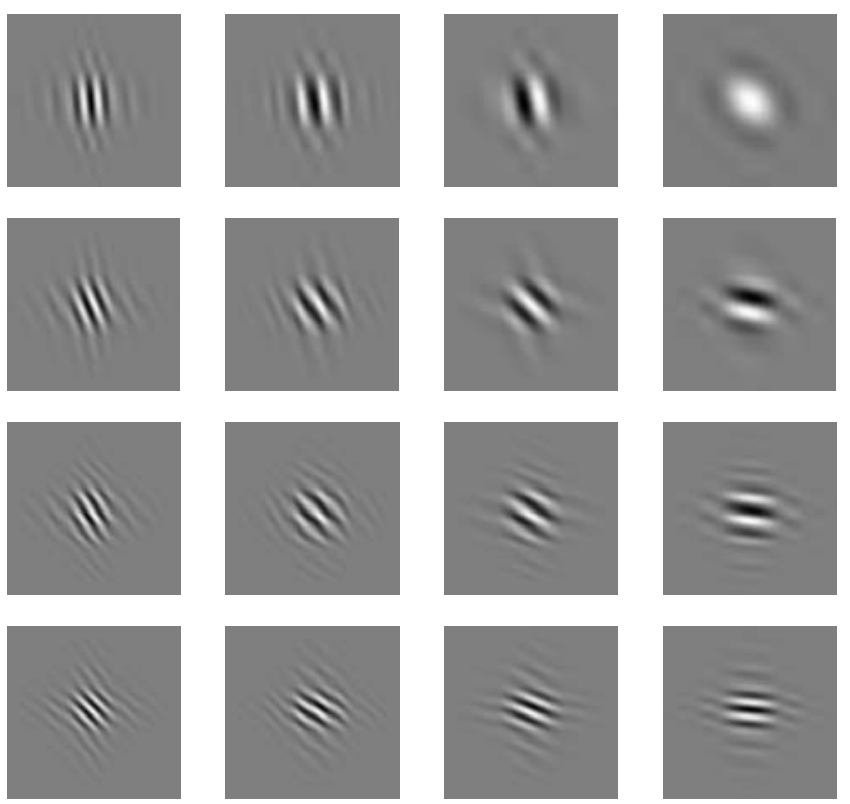

Fig. 10. Resulting directional wavelets of the 4-band dualtree wavelet transform. Half the wavelets are illustrated (the other wavelets lie perpendicular to the illustrated wavelets).

that we do this only for even $i$ ). Thus, the new decomposition may be expressed as $\hat{V}_{i}=\hat{V}_{i+2} \oplus \hat{W}_{i+2} \oplus \hat{W}_{i+1}^{0} \oplus \hat{W}_{i+1}^{1}$ for even $i$. Consequently, it can be stated that our construction employs approximation spaces $V_{i}$ related to the spaces of the 2-band basis as $V_{i}=\hat{V}_{2 i}$. This is clearly a special case of the construction in (14) and (15). Thus, it is likely that there exist superior FIR filters in terms of having shorter support for the same number of vanishing moments and providing a better approximation to Hilbert transform wavelet pairs, which may be obtained by a procedure that does not place any constraints on the structure of the 4-channel filter bank similar to the case of the real $M$-band DWT as in $[13,12]$. However, an advantage of our construction is the ease of obtaining filters which rely on the 2-band DT- $\mathbb{C W T}$ for which already a body of literature exists $[6,4,10]$ (also see $[5,11]$ for an overview).

\section{CONCLUSION}

We have extended the 2-band DT-CWT to a dual-tree wavelet packet transform and derived a 4-bank DT-CWT. This approach puts into use the existing knowledge for the 2-band case and we are able to obtain FIR filters with good approximation properties. We have also extended the transform to 2$\mathrm{D}$ and showed that the transform achieves a directional analysis. The introduced transforms are $2 X$ overcomplete in 1D and $2 \mathrm{X}$ or $4 \mathrm{X}$ overcomplete in $2 \mathrm{D}$, depending on the choice of the $2 \mathrm{D}$ implementation.

\section{REFERENCES}

[1] C. Chaux, L. Duval, and J.-C. Pesquet. Hilbert pairs of M-band orthonormal wavelet bases. In Proc. Eur. Sig. and Image Proc. Conf., 2004.

[2] C. Chaux, L. Duval, and J.-C. Pesquet. Image analysis using a dual-tree M-band wavelet transform. IEEE Trans. on Image Processing, 15(8):2397-2412, August 2006.

[3] A. Jalobeanu, L. Blanc-Féraud, and J. Zerubia. Satellite image deconvolution using complex wavelet packets. In Proc. IEEE Int. Conf. on Image Processing (ICIP), 2000.

[4] N. G. Kingsbury. Image processing with complex wavelets. Philos. Trans. R. Soc. London A, Math. Phys. Sci., 357(1760):2543-2560, September 1999.

[5] N. G. Kingsbury. Complex wavelets for shift invariant analysis and filtering of signals. J. of Appl. and Comp. Harm. Analysis, 10(3):234-253, May 2001.

[6] N. G. Kingsbury. Design of q-shift complex wavelets for image processing using frequency domain energy minimization. In Proc. IEEE Int. Conf. on Image Processing (ICIP), 2003.

[7] S. Mallat. A Wavelet Tour of Signal Processing. Academic Press, 1998.

[8] H. Özkaramanlı and R. Yu. On the phase condition and its solution for Hilbert transform pairs of wavelet bases. IEEE Trans. Signal Processing, 51(12):3293-3294, December 2003.

[9] I. W. Selesnick. Hilbert transform pairs of wavelet bases. IEEE Signal Processing Letters, 8(6):170-173, June 2001.

[10] I. W. Selesnick. The design of approximate Hilbert transform pairs of wavelet bases. IEEE Trans. Signal Processing, 50(5):1144-1152, May 2002.

[11] I. W. Selesnick, R. G. Baraniuk, and N. G. Kingsbury. The dual-tree complex wavelet transform - A coherent framework for multiscale signal and image processing. IEEE Signal Processing Magazine, 22(6):123-151, November 2005.

[12] P. Steffen, P. N. Heller, R. A. Gopinath, and C. S. Burrus. Theory of regular $m$-band wavelet bases. IEEE Trans. Signal Processing, 41(12):3497-3511, December 1993.

[13] G. V. Welland and M. Lundberg. Construction of compact p-wavelets. Constructive approximation, 9(23):347-370, June 1993. 